\title{
LOCAL HALF MOUFANG QUADRANGLES
}

\author{
KOEN THAS
}

\begin{abstract}
AвSTRACT. In this paper, we show that if every root of a finite generalized quadrangle containing a fixed point is Moufang, then every dual root containing it is also Moufang. As a corollary, we obtain a new proof of the half Moufang theorem. This says that finite half Moufang quadrangles are Moufang.
\end{abstract}

\section{INTRODUCTION}

A famous and old question of Tits (going back to the 1960s) asks for the existence of locally finite generalized polygons: these are generalized polygons (spherical buildings of rank 2) which, up to duality, have a finite number of points on a line and an infinite number of lines incident with a point. For generalized polygons of odd gonality at least 3 , both numbers are equal, and so no such polygons exist. The group theoretical analogon of this question wonders about the existence of half Moufang generalized polygons which are not Moufang. This question only makes sense for generalized polygons of even gonality; for generalized $(2 k+1)$-gons $(k \geq 1$ a positive integer), there is no distinction between half Moufang and Moufang since there only is one type of root.

We will not survey the Moufang condition, which is the central group theoretical property in the theory of rank 2 buildings, nor its importance, here. Rather, we refer to the many surveys and papers which are vailable, such as [1, 2], [9], [3] or [6], and especially the celebrated work [10] which classifies Moufang polygons.

For the rest of the note, we consider the (most important) case of generalized 4-gons, or generalized quadrangles.

In 1991 Thas, Payne and Van Maldeghem [5] finally succeeded in showing that finite half Moufang generalized quadrangles are indeed Moufang. The proof is not direct, and not elementary, in the sense that they use a classification of half Moufang GQs which can be found in [3] (see also the references therein), which amounts to stating that finite half Moufang GQs either arise from classical groups, or have some particular combinatorial property. Since classical quadrangles are known to be Moufang, the main part of [5] is then concerned with handling the combinatorial part.

In [4], Tent obtained the same result without the assumption that the quadrangle be finite. She obtains a direct proof without invoking any classifcation result, but of course uses the global half Moufang condition. (Note that a crucial lemma of Van Maldeghem was used in [4].)

2000 Mathematics Subject Classification. 05B25, 20B10, 20B25, 20E42, 51E12.

The author is partially supported by the Fund for Scientific Research - Flanders (Belgium). 
In this short note, we only assume that a finite GQ is half Moufang "at a point", and then show that it is necessarily Moufang at that point. The half Moufang theorem easily follows. The proof is direct, and uses no classification result whatsoever. And on the other hand, contrary to the "infinite proof", we do not use global Moufang conditions. In fact, the only thing we do is put a part of the proof of [5] and of [7] together to obtain the main result. But it does the trick.

To the author's knowledge, it is the first time for quadrangles (or generalized polygons of higher gonality) that, without any extra combinatorial or group theoretical assumptions, additional (general) Moufang conditions are forced from only local (general) Moufang conditions.

The ideas of the present note were found during the preparation of a final part of an advanced course on Incidence Geometry, taught at Ghent University in October - December of 2010.

Acknowledgment. The author thanks an anonymous referee for providing him several very useful suggestions.

\section{SETTING}

Let $\Gamma$ be a thick generalized quadrangle (GQ). It is a rank 2 geometry $\Gamma=$ $(\mathscr{P}, \mathscr{B}, \mathbf{I})$ (where we call the elements of $\mathscr{P}$ "points" and those of $\mathscr{B}$ "lines") such that the following axioms are satisfied:

(a) there are no ordinary digons and triangles contained in $\Gamma$;

(b) each two elements of $\mathscr{P} \cup \mathscr{B}$ are contained in an ordinary quadrangle;

(c) there exists an ordinary pentagon.

It can be shown that there are exist constants $s$ and $t$ such that each point is incident with $t+1$ lines and each line is incident with $s+1$ points. We say that $(s, t)$ is the order of $\Gamma$. Note that an ordinary quadrangle is just a (necessarily thin) GQ of order $(1,1)$ - we call such a subgeometry also "apartment" (of $\Gamma$ ).

Let $A$ be an apartment of a GQ $\Gamma$. A root $\gamma$ of $A$ is a set of 5 different elements $e_{0}, \ldots, e_{4}$ in $A$ such that $e_{i} \mathbf{I} e_{i+1}$ (where the indices are taken in $\{0,1,2,3,4\}$ ), and $e_{0}, e_{4}$ are the extremal elements of $\gamma$. There are two types of roots, depending on whether the extremal elements are lines or points; in the second case we speak of dual roots to make a distinction between the types. Also, a (dual) root $\gamma$ without its extremal elements - the interior of $\gamma$ - is denoted by $\dot{\gamma}$ and called (dual) i-root.

If $\mathscr{M}$ is a subgeometry of $\Gamma$, by $\operatorname{Aut}(\Gamma)^{[\mathscr{M}]}$ we denote the subgroup of the automorphism group $\operatorname{Aut}(\Gamma)$ of $\Gamma$ which fixes every line incident with a point of $\mathscr{M}$ and every point incident with a line of $\mathscr{M}$. Now a (dual) root $\gamma$ is Moufang if $\operatorname{Aut}(\Gamma)^{[\dot{\gamma}]}$ acts transitively on the apartments containing $\gamma$. In fact, $\operatorname{Aut}(\Gamma)^{[\dot{\gamma}]}=$ : $A(\dot{\gamma})$ then acts sharply transitively on these apartments. Once a (dual) root $\gamma$ is Moufang, all (dual) roots with interior $\dot{\gamma}$ are also Moufang, with respect to the same group $A(\dot{\gamma})$. (The latter groups are uniquely defined by $\dot{\gamma}$ and the Moufang property.) In a natural way, we also use the terms "Moufang i-root" and "dual Moufang i-root", and the elements of $A(\dot{\gamma})$ are called root-elations throughout. 
Now $\Gamma$ is half Moufang if all roots or all dual roots are Moufang. It is Moufang if all roots and dual roots are.

\section{Local half Moufang theorem}

We start with a lemma which does not rely on finiteness. It is similar to a lemma of [5] where it is obtained in the finite case (the proof also works in general). For this purpose, an elation generalized quadrangle (EGQ) is a GQ $\Gamma$ containing some point $x$ such that there exists a subgroup $H$ of $\operatorname{Aut}(\Gamma)^{[\{x\}]}$ which acts sharply transitively on the points not collinear with $x$. We call $H$ elation group, and also write $\Gamma^{x}$ to specify the GQ with elation point.

Lemma 3.1. Let $\Gamma^{x}$ be an EGQ with elation group $H$. Suppose $(x, L, u)=\rho$ and $(x, M, v)=\mu$ are Moufang $i$-roots, with $L \neq M$ lines on $x$, and such that $A(\rho) \leq H$, $A(\mu) \leq H$. Then $(L, x, M)=\tau$ is a dual Moufang i-root with $A(\tau) \leq H$.

Proof. Let $z \in\{u, v\}^{\perp} \backslash\{x\}$, and let $x_{i}, i \in I$, be the different points of $\{z, x\}^{\perp}$ (for some index set $I$ containing symbols 0,1 and bijective with the set of lines incident with $x$ ), in such a way that $u=x_{0}$ and $v=x_{1}$. Let $z x_{k}=W_{k}$, and $x x_{k}=L_{k}$ for all $k \in I$. Define $H_{i}=H_{W_{i}}$ and $H_{i}^{*}=H_{x_{i}}$ for $i \in I$. Then for each $j \in I$

$$
H_{j}^{*}, \quad H_{j} H_{k} \backslash H_{j}, \quad k \in I \backslash\{j\}
$$

defines a partition of $H$. For, let $\kappa \in H^{\times}$, and consider $z^{\kappa}$. If $\operatorname{proj}_{L_{j}} z^{\kappa}=\operatorname{proj}_{L_{j}} z=$ $x_{j}$, then $\kappa \in H_{j}^{*}$, so suppose now that $\operatorname{proj}_{L_{j}} z^{\kappa}$ is different from $\operatorname{proj}_{L_{j}} z$. Put $\operatorname{proj}_{z^{k}} L_{j}=W$, and $\operatorname{proj}_{W^{\prime}} z=z^{\prime}$. Let $r \in I$ be such that $z z^{\prime}=W_{r}$, and let $\phi \in H$ map $z$ to $z^{\prime}$. Then $\phi$ maps $W_{r}=\operatorname{proj}_{z} L_{r}$ to $W_{r}=\operatorname{proj}_{z^{\prime}} L_{r}$ and $W_{j}=\operatorname{proj}_{z} L_{j}$ to $W=\operatorname{proj}_{z^{\prime}} L_{j}$, while $\kappa$ maps $W_{j}=\operatorname{proj}_{z} L_{j}$ to $W=\operatorname{proj}_{z^{k}} L_{j}$. Thus $\phi \in H_{r}$ and $\kappa \in H_{j} \phi \subseteq H_{j} H_{r}$, and hence

$$
H=H_{j}^{*} \bigcup\left(\cup_{k \in I \backslash\{j\}} H_{j} H_{k} \backslash H_{j}\right) .
$$

The fact that we indeed have a partition now follows from the following properties (which are well-known to hold, cf. [8]):

- $H_{v}^{*} \cap H_{\mu}=\{\mathbf{1}\}$ for all distinct $v, \mu \in I$;

- $H_{v} H_{\mu} \cap H_{\ell}=\{\mathbf{1}\}$ for all distinct $v, \mu, \ell \in I$.

Put $j=0$ and let $y \mathbf{I} x u$, with $y \neq x$. We observe that $H_{0}$ fixes $L$ pointwise since the assumption $A(\rho) \leq H$ implies $H_{0}=H_{W_{0}}=A(\rho)$. For $k \neq 0, k \in I$, the only elements in $H_{0} H_{k}$ fixing a point of $L \backslash\{x\}$ are in $H_{0}$, since $H_{0}$ fixes $L$ pointwise, and $H_{k}$ acts sharply transitively on the latter set. By $(\mathbb{1})$, it follows that $H_{y} \leq H_{0}^{*}=H_{y}$, the equality coming from the fact that $H_{y}$ and $H_{0}^{*}$ are conjugate in $H$, and so $H_{0}^{*}$ fixes $L$ pointwise since $y$ was arbitrary on $L$. Hence $H_{1}^{*}$ also fixes $M$ pointwise, and the group $H_{u, v}=H_{0}^{*} \cap H_{1}^{*}$, which acts sharply transitively on $\{u, v\}^{\perp} \backslash\{x\}$, fixes $L$ and $M$ pointwise (and, of course, $x$ linewise). So $(L, x, M)=\tau$ is a dual Moufang i-root with group

$$
A(\tau)=H_{u, v} \leq H
$$


KOEN THAS

Corollary 3.2. Let $\Gamma$ be a generalized quadrangle such that all i-roots containing some fixed point $x$ are Moufang. If the group $H$ generated by all the corresponding rootelations makes $\Gamma^{x}$ an EGQ with elation group $H$, then all dual i-roots containing $x$ are also Moufang.

Proof. Immediate by the preceding lemma.

We now prove the main result.

Theorem 3.3. Let $\Gamma$ be a finite generalized quadrangle such that all i-roots containing some fixed point $x$ are Moufang. Then all dual i-roots containing $x$ are also Moufang.

Proof. Define the group $H$ as in the statement of the previous corollary. By Theorem 1.1 of $[7], \Gamma^{x}$ is an EGQ with elation group H. So by Corollary 3.2 , the theorem follows.

At the moment, no infinite version of [7, Theorem 1.1] is available.

3.1. The half Moufang theorem. Passing to the global result now goes very smoothly.

Let $\Gamma$ be a finite half Moufang GQ. Suppose without loss of generality that all roots are Moufang. Take any point $z$; then by Theorem 3.3 all dual roots on $z$ are Moufang. Since $z$ was arbitrary, it follows that $\Gamma$ is Moufang.

3.2. The converse. One might be tempted to try for a converse of Theorem 3.3. namely, to obtain that if all dual i-roots on some point of a GQ are Moufang, then also all i-roots on that point are. Or, even stronger, one might wonder whether the group generated by the dual root-elations in this setting contains "complete groups" of root-elations corresponding to any i-root on the point.

Neither is true. For the second converse, recall that a center of symmetry is a point $x$ such that any dual i-root $\tau$ containing $x$ is Moufang, and for which $A(\tau)$ is independent of the choice of $\tau$. That is, each element of $A(\tau)$ fixes $x^{\perp}$ elementwise. Now if $x$ is as such, then

$$
A(x):=\langle A(\tau)| \tau \text { is a dual i-root on } \mathrm{x}\rangle=A(\rho),
$$

where $\rho$ is any dual i-root containing $x$, and many examples of finite and infinite GQs exist with centers of symmetry (one being defined below).

The first converse is more subtle; although we have shown that even if all dual roots on a point $x$ are Moufang, $A(x)$ does not necessarily contain nontrivial rootelations, it still could be that all i-roots containing $x$ must be Moufang (that is, that both conditions cannot live without each other). By a recent observation of the author which is contained in [8], we will show that this is also not true. 
Let $q=2^{e}$ be an odd power of 2 , and let $\sigma \in \operatorname{Aut}\left(\mathbb{F}_{q}\right)$ be such that $\sigma^{2}=2$. Define the map

$$
f: \mathbb{F}_{q}^{2} \rightarrow \mathbb{F}_{q}:(a, b) \rightarrow a^{\sigma+2}+a b+b^{\sigma} .
$$

The Tits ovoid of $\operatorname{PG}(3, q)$ is given by

$$
\mathscr{O}=\{(0,1,0,0)\} \cup\left\{(1, f(a, b), a, b) \mid a, b \in \mathbb{F}_{q}\right\} .
$$

We suppose that $e>1$, so that $\mathscr{O}$ is not an elliptic quadric. Let $\Gamma=\mathbf{T}_{3}(\mathscr{O})$ be the corresponding TGQ of order $\left(q, q^{2}\right)$ with translation point $(\infty)[6]$, and let $L \mathbf{I}(\infty)$ be the line of $\Gamma$ corresponding with the point $(0,1,0,0)$. (Note that $\operatorname{Aut}(\Gamma)$ acts transitively on the lines incident with $(\infty)$.) Embed PG $(3, q)$ as a hyperplane in PG $(4, q)$, by

$$
(x, y, z, w) \rightarrow(0, x, y, z, w) .
$$

Define collineations $\theta(a, b, c, d, e):(u, x, y, z, w) \rightarrow(u, x, y, z, w)[a, b, c, d, e]$ of $\operatorname{PG}(4, q)$, with $a, b, c, d, e \in \mathbb{F}_{q}$, where

$$
[a, b, c, d, e]=\left(\begin{array}{ccccc}
1 & 0 & c & d & e \\
0 & 1 & f(a, b) & a & b \\
0 & 0 & 1 & 0 & 0 \\
0 & 0 & a^{\sigma+1}+b & 1 & a^{\sigma} \\
0 & 0 & a & 0 & 1
\end{array}\right)
$$

Then $G=\left\{\theta(a, b, c, d, e) \mid a, b, c, d, e \in \mathbb{F}_{q}\right\}$ is a group of order $q^{5}$ with binary operation

$$
\begin{aligned}
& {[a, b, c, d, e]\left[a^{\prime}, b^{\prime}, c^{\prime}, d^{\prime}, e^{\prime}\right]} \\
& =\left[a+a^{\prime}, b+b^{\prime}+a a^{\prime \sigma}, c+c^{\prime}+d\left(a^{\prime \sigma+1}+b^{\prime}\right)+e a^{\prime}, d+d^{\prime}, e+e^{\prime}+d a^{\prime \sigma}\right],
\end{aligned}
$$

so that $\Gamma^{D}$ is an EGQ with elation group $G$ and elation point $x$ corresponding to $L$ [8]. Now define for $t \in \mathbb{F}_{q}$ :

(10) $A(t)=\left\{[a, b, t f(a, b), t a, t b] \mid a, b \in \mathbb{F}_{q}\right\}, A^{*}(t)=\left\{[a, b, c, t a, t b] \mid a, b, c \in \mathbb{F}_{q}\right\}$

and

$$
A(\infty)=\left\{[0,0,0, d, e] \mid d, e \in \mathbb{F}_{q}\right\}, A^{*}(\infty)=\left\{[0,0, c, d, e] \mid c, d, e \in \mathbb{F}_{q}\right\} .
$$

Then $\mathbf{F}=\left\{A(t) \mid t \in \mathbb{F}_{q} \cup\{(\infty)\}\right\}$ and $\mathbf{F}^{*}=\left\{A^{*}(t) \mid t \in \mathbb{F}_{q} \cup\{(\infty)\}\right\}$ define a Kantor family of $G$ associated to the point $\langle(1,0,0,0,0),(0,1,0,0,0)\rangle$ of $\Gamma^{D}$ [8].

Proposition $3.4\left(\mathrm{~K}\right.$. Thas [8]). $\quad$ (i) $A^{*}(\infty)$ and $A(\infty)$ are elementary abelian and $A^{*}(\infty) \unlhd G$, so that $A^{*}(\infty)$ fixes $[A(\infty)]$ pointwise.

(ii) For $t \in \mathbb{F}_{q}, A^{*}(t)$ and $A(t)$ are nonabelian of exponent 4; moreover, for $t \neq t^{\prime}$ in $\mathbb{F}_{q}, A^{*}(t) \cong A^{*}\left(t^{\prime}\right)$ and $A(t) \cong A\left(t^{\prime}\right)$. Also, no $A^{*}(t)$ is normal in $G$.

(iii) $G$ is the complete set of elations about $x$.

(iv) $x$ is a center of symmetry, and the symmetries are contained in $G$.

By (iv), all dual i-roots on $x$ are Moufang. Also, any root-elation with i-root containing $x$ must be in $G$, by (iii). But then by (ii) no i-root containing a line 
corresponding to $t \in \mathbb{F}_{q}$ can be Moufang.

So in some sense Theorem 3.3 is the best possible from the local point of view.

\section{REFERENCES}

[1] P. Fong AND G. M. SeItz. Groups with a (B, N)-pair of rank 2. I, Invent. Math. 21 (1973), 1-57.

[2] _ Groups with a (B, N)-pair of rank 2. II, Invent. Math. 24 (1974), 191-239.

[3] S. E. Payne and J. A. Thas. Finite Generalized Quadrangles, Research Notes in Mathematics 110, Pitman Advanced Publishing Program, Boston/London/Melbourne, 1984.

[4] K. Tent. Half Moufang implies Moufang for generalized quadrangles, J. reine Angew. Math. 566 (2004), 231-236.

[5] J. A. Thas, S. E. Payne and H. Van Maldeghem. Half Moufang implies Moufang for finite generalized quadrangles, Invent. Math. 105 (1991), 153-156.

[6] J. A. Thas, K. Thas and H. Van Maldeghem. Translation Generalized Quadrangles, Series in Pure Mathematics 26, World Scientific Publishing Co. Pte. Ltd., Hackensack, NJ, 2006.

[7] K. Thas. Solution of a question of Knarr, Proc. Amer. Math. Soc. 136 (2008), 1409-1418.

[8] _ A Course on Elation Quadrangles, 135 pp., European Math. Soc., To appear.

[9] J. Tits. Buildings of Spherical Type and Finite BN-Pairs, Lecture Notes in Math. 386, Springer, Berlin, 1974 .

[10] J. Tits and R. M. Weiss. Moufang Polygons, Springer Monographs in Mathematics, SpringerVerlag, Berlin, 2002.

Ghent University, Department of Mathematics, Krijgslaan 281, S25, B-9ooo Ghent, Belgium

E-mail address: kthas@cage. UGent. be 